\title{
The determination of the GX 339-4's mass based on its 2010 outburst
}

\author{
Tao Chen \\ CEA \Saclay, Bât. 709, Orme des Merisiers \\ 91191 Gif sur Yvette Cedex, France \\ email: tao.chen@cea.fr
}

\begin{abstract}
In this paper we investigate the quasi periodic oscillation (QPO) behavior of the black hole candidate GX 339-4 during its 2010 outburst using RXTE/PCA data. We perform spectral and timing analysis of the observations, where the QPOs are observed. We analyze the relationship between the centroid frequency of QPO and the spectral parameters. The correlation of spectral and timing properties can be used to estimate the mass of black hole with the scaling method. Using this method we estimate a mass of $7.5 \pm 0.8 M_{\odot}$ of GX 339-4.
\end{abstract}

Keywords. GX 339, QPO, Black Hole

\section{Introduction}

The determination of the mass of Galactic black holes (BHs) is one of the most important tasks in modern astronomy. Since it can constrain the maximum mass of a neutron star and the minimum mass of a BH. The knowledge of the $\mathrm{BH}$ mass distribution of the Galaxy can also provide important clues on stellar evolution. In particular, the mass of $\mathrm{BH}$ can be estimated by the correlation between low-frequency quasi-periodic oscillations (LFQPOs, which observed during low-hard and intermediate states and $\leqslant 10 \mathrm{~Hz}$ ) and photon index of the power-law spectral component (Shaposhnikov \& Titarchuk 2006).

\section{Observations \& Data Analysis}

We analyzed 28 the RXTE observations of GX 339-4 based on its 2010 outburst. For timing analysis, the light curves were extracted from event data using $2-50 \mathrm{KeV}$ energy bands. Power spectra were produced for 256 seconds interval with 0.015625 seconds time resolution. For spectral analysis, the PCA Standard 2 mode (STD2) in energy range 2.5-45.0 keV was used for extraction of the energy spectra. In order to account for residual uncertainties in the instrument calibration a systematic error of 0.8 percent was added to spectra.

For spectral analysis, we fit the energy spectrum where the clear LFQPOs were observed. During the low-hard state, the spectra can be well fitted by the model of exponentially cut-off power law reflected from ionized matter (pexriv) and a Simple Gaussian line profile (gaussian). For the intermediate state we use a simple photon power law (powerlaw), a disk blackbody (diskbb) and a Gaussian (gaussian). For both case the photonelectric absorption was frozen to $0.51 \times 10^{22} \mathrm{~cm}^{-2}$ (Kong et al. 2000). The state transition of this source was reported by Motta et al. (2010). The photon indices are very important for determination the mass. The change of photon indexes is shown in Fig. 1.

For the timing analysis, the frequencies of QPOs are obtained by fitting the clear peak on power spectra with a Lorentzian. For each peak we use only one Lorentzian. The 
change of QPO frequencies is shown in Fig. 2. Comparing this figure with Fig. 1, there are some similar tendency on these two figures. This can be clearly seen in Fig. 3.

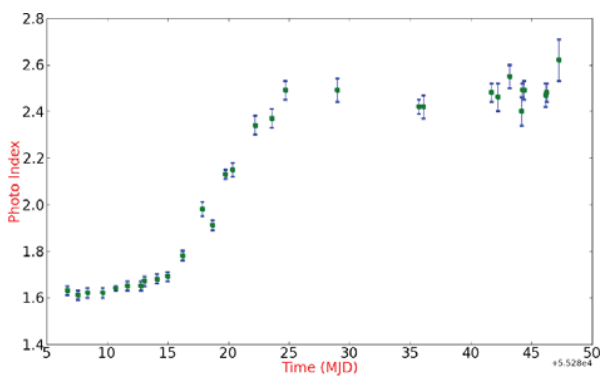

Figure 1. The change of photon indexes with time.

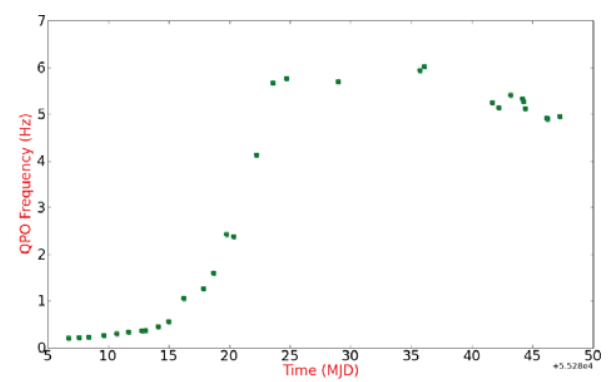

Figure 2. The change of QPO frequencies with time.

\section{Scaling method \& Conclusion}

Shaposhnikov \& Titarchuk (2006) have shown that the correlation between photon index and QPO frequency can be used to estimate BH mass: First of all, we fit this correlation with an analytical function (see red line on Fig. 3): $f(\nu)=A-D B \ln \left[\exp \left(\frac{\nu_{t r}-\nu}{D}\right)+1\right]$.

For determination of the BHs mass $M$, the parameter $B$ should be knew. It can be obtained by fitting of correlation between photon index and QPO frequency with the above function. Next step is to compare this value with a standard source, which has a well-known mass. GRO J1655-40 (which commonly used for this method as its mass is known by optical method) was used for this purpose. Finally, by analysis spectral and timing properties of GX 3394 , based on its 2010 outburst, we found that the photon index and QPO frequency

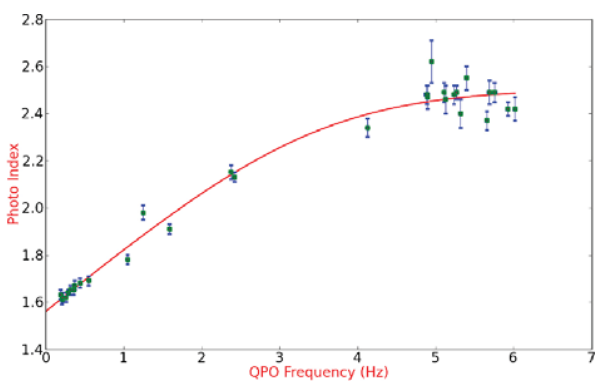

Figure 3. The correlation between photon index and QPO frequency. showed a strong correlation. Using scaling method based on this correlation we estimated the mass of GX 339-4: $M_{G X 339}=B_{G X 339} \frac{M_{J 1655}}{B_{J 1655}}=7.5 \pm 0.8 M_{\odot}$. This result consistent with previous prediction $M_{G X 339} \geqslant 6 M_{\odot}($ Hynes et al. 2003).

\section{Acknowledgments}

This work is funded by the Europe Communitys Seventh Framework Programme (FP7/2007-2013) under grant agreement number ITN 215212 Black Hole Universe.

\section{References}

Hynes R. I. et al. 2003, ApJ, 583, L95

Kong A. K. H. et al. 2000, MNRAS, 312, L49

Motta S. et al. 2010, ATel \#2545

Shaposhnikov N. \& Titarchuk L. 2006, ApJ, 699, 453 